QUARTERLY OF APPLIED MATHEMATICS

VOLUME LXVI, NUMBER 1

MARCH 2008, PAGES 69-80

S 0033-569X(07)01078-0

Article electronically published on December 18, 2007

\title{
AN ANALYSIS OF THE PARAXIAL WAVE EQUATION
}

\author{
BY \\ PETER A. MCCOY (Department of Mathematics, U.S. Naval Academy, Annapolis, Maryland \\ 21402-5002) \\ AND
}

REZA MALEK-MADANI (Department of Mathematics, U.S. Naval Academy, Annapolis, Maryland 21402-5002)

\begin{abstract}
Function theoretic methods are used to characterize solutions of the paraxial wave equation in an isotropic homogeneous medium in 3-space. A new class of function theoretic solutions whose singularities are manifested as sectionally analytic functions is constructed via integral transforms.
\end{abstract}

1. Introduction. The paraxial wave equation (PWE) is of fundamental importance in the modeling of an optical beam propagating through an isotropic homogeneous medium. The inclusion of higher-order atmospheric effects such as thermal blooming, plasma jets, beam cavitation, and motion of the medium are complex time-dependent phenomena that require non-linear methods in their modelling and simulation. For nonlinear phenomena, see $[5,17,18]$.

The aim of this paper is to discuss some of the characteristics of an asymmetric beam propagating through a uniform medium with zero conductivity in the absence of free charge. The higher-order effects mentioned above will be considered in subsequent papers. The approach used here employs both a normal mode analysis and function theoretic methods $[3,4,7]$. Normal mode method is one of the best ways to determine numerical stability of a solution. The function theoretic method has demonstrated great utility in the analysis and characterization of solutions to a broad range of problems in mathematical physics (see [3, 4], [6]-[8], [11]-[15], [20]). This paper is the first application of these methods in this venue and leads to some new and interesting results.

Reciprocal integral transforms based on normal mode expansions are constructed that link analytic solutions of the PWE with associated analytic functions of two complex variables. Analytic continuation of these formulae allows characterization of solutions that are analytic in a half-space as well as the construction of a new class of function theoretic solutions with singularities. The singularities of these solutions demonstrate a novel and radical departure in behavior from the singularities of analytic solutions

Received May 31, 2006.

2000 Mathematics Subject Classification. Primary 35L05, 35Q60. 
of elliptic partial differential equations. Analytic solutions of elliptic partial differential equations exhibit a "pole-like" behavior in a neighborhood of a singularity, whereas a singularity of a solution to the PWE is manifested in terms of a sectionally analytic function that is adjoined to the solution as the singularity is included in the analytic continuation of the associate.

2. Background. We begin our investigation of optical beam propagation with Maxwell's equations

$$
\begin{array}{ll}
(1 a) \vec{\nabla} \cdot \vec{E}=0 & (1 b) \vec{\nabla} \cdot \vec{B}=0 \\
(1 c) \vec{\nabla} \times \vec{E}=-1 / c \partial \vec{B} / \partial t & (1 d) \vec{\nabla} \times \vec{B}=1 / c \partial \vec{E} / \partial t
\end{array}
$$

which are known respectively as Coulomb's Law, Gauss's Law for magnetism, Faraday's Law, and Ampère's Law. The electric field is designated by $\vec{E}=\vec{E}(x, y, z, t)$ and the magnetic field by $\vec{B}=\vec{B}(x, y, z, t)$.

The derivation of the paraxial wave equation starts with the reduction of Maxwell's system to the wave equation. We take the curl of Faraday's Law,

$$
\vec{\nabla} \times \vec{\nabla} \times \vec{E}=-1 / c \partial(\vec{\nabla} \times \vec{B}) / \partial t,
$$

apply the vector identity $\vec{\nabla} \times \vec{\nabla} \times \vec{E}=\vec{\nabla}(\vec{\nabla} \cdot \vec{E})-\nabla^{2} \vec{E}$, and then Coulomb's Law to find that

$$
\nabla^{2} \vec{E}=1 / c \partial(\vec{\nabla} \times \vec{B}) / \partial t .
$$

Subsequent application of Ampère's Law yields the wave equation

$$
\left[\nabla^{2}-1 / c^{2} \partial^{2} / \partial t^{2}\right] \vec{E}(x, y, z, t)=\overrightarrow{0}
$$

A remark concerning the coordinates is in order. The optical axis and the $z$-axis are aligned. The origin is placed at the focal point where the beam's waist spot size is minimum. The reference plane $z=0$ ( $x y$-plane) passes through the focal point and is transverse to the optical axis.

Consider the phasor amplitude $H$ of the component $E_{z}$ of $\vec{E}$ along the $z$-axis. The wave equation becomes time-independent under the change of variables

$$
E_{z}(x, y, z, t)=H(x, y, z) e^{-i \omega t},
$$

which leads to the Helmholtz equation

$$
\left[\frac{\partial^{2}}{\partial x^{2}}+\frac{\partial^{2}}{\partial y^{2}}+\frac{\partial^{2}}{\partial z^{2}}+k^{2}\right] H=0 .
$$

The wave number $k=\omega / c=2 \pi / \lambda$, where $\lambda$ is the wave length.

It is generally known that the transverse variation in the amplitude is slow as compared to its variation in the $z$-direction. The rapidly varying component in the $z$-direction is separated out by removing the primary propagation factor by the transformation

$$
H(x, y, z)=F(x, y, z) e^{-i k z},
$$


where the function $F$ represents the spatial modulation of the plane wave. This transformation orients the motion in the direction of increasing the $z$ coordinate and yields the parsed Helmholtz equation

$$
\left[\frac{\partial^{2}}{\partial x^{2}}+\frac{\partial^{2}}{\partial y^{2}}+\frac{\partial^{2}}{\partial z^{2}}-2 i k \frac{\partial}{\partial z}\right] F=0
$$

which is the precursor of the PWE. The reader should note that the above two transformations can be accomplished by a single change of variables. However, the physical motivation is concealed in the process.

The study of the variation of the modulation in terms of the $z$-variable requiring asymptotic analysis is an open problem. We follow the traditional method as found in [16, p. 627-629]. Consider the plane wave

$$
H(x, y, z)=e^{-i k(x \sin \theta+y \sin \theta+z \cos \theta)}
$$

advancing along the $z$-axis in $E^{3}$ where $\theta$ is the colatitude, i.e., the wave is traveling at an angle $\theta$ relative to the optical axis. The wave is oriented in the $z$-direction by the above change of dependent variables from which we determine that the amplitude is

$$
F(x, y, z)=e^{-i k(x \sin \theta+y \sin \theta-z(1-\cos \theta))} .
$$

The paraxial approximation is introduced for small angles $\theta$. The fact that the amplitude is a slowly varying function of the range is confirmed by the approximations

$$
\begin{aligned}
& F(x, y, z) \approx e^{-i k\left(x \theta+y \theta+z \theta^{2} / 2\right)}, \\
& \frac{2 i k}{F} F_{z}(x, y, z)=2 k^{2}(1-\cos \theta) \approx k^{2} \theta^{2}, \\
& \frac{1}{F} F_{x x}=\frac{1}{F} F_{y y}(x, y, z)=(-i k \sin \theta)^{2} \approx-k^{2} \theta^{2}, \\
& \frac{1}{F} F_{z z}(x, y, z)=(-i k(1-\cos \theta))^{2} \approx-k^{2} \theta^{4} / 4 .
\end{aligned}
$$

Scale analysis yields the estimate

$$
\left|\frac{1}{F} \frac{\partial^{2} F}{\partial z^{2}}\right|<<\min \left\{\left|\frac{2 k}{F} \frac{\partial F}{\partial z}\right|,\left|\frac{1}{F} \frac{\partial^{2} F}{\partial x^{2}}\right|,\left|\frac{1}{F} \frac{\partial^{2} F}{\partial y^{2}}\right|\right\},
$$

which is valid to within an error of $O\left(\theta^{2} / 4\right)$ as $\theta \rightarrow 0$. For example, the maximum error in the approximation is at most $1.7 \%$ in a cone of opening $\pi / 12$ radians about the optical axis.

Implementing this estimate gives the paraxial wave equation

$$
\left[\nabla_{t}^{2}-2 i k \frac{\partial}{\partial z}\right] F=0
$$

where $\nabla_{t}^{2}=\vec{\nabla}_{t} \cdot \vec{\nabla}_{t}$ is the transverse Laplacian and $\vec{\nabla}_{t}=(\partial / \partial x, \partial / \partial y)$ is the transverse gradient. There are two forms of solution for this equation. The first represents the so-called search light or Gaussian beam propagating through space:

$$
\Psi\left(x^{2}+y^{2}, z\right)=\frac{w(0)}{w(z)} \exp \left\{-i \arctan \left(\frac{z}{z_{R}}\right)\right\} \exp \left\{\phi\left(x^{2}+y^{2}, z\right)\right\}
$$


where $\phi(\xi, z)=-\xi\left\{i k / 2 r(z)+1 / w^{2}(z)\right\}$. The waist spot size of the beam is given by $w(z)=w_{o} \sqrt{1+\left(z / z_{R}\right)^{2}}$ [16, p. 664-665]. The principal branches of radicals are taken. The Rayleigh range $z_{R} \equiv \pi w_{o}^{2} / \lambda$ generally marks the transition between the near-field and the far-field patterns of the beam. This parameter can be viewed as the distance from the focal point to the point where the beam's diameter has expanded by a factor of $\sqrt{2}$ of its minimum waist spot size $w_{o}[16$, p. 668-669]. The radius of curvature $r(z)=z+z_{R}^{2} / z$. The source is located to the left of the reference plane at the point $\left(0,0,-z_{S}\right)$, where $z=-z_{S}\left(z_{R} \leq z_{S}\right)$ is the plane containing the source.

We are interested in the structure of higher-order Gaussian beams. The standard protocol (see [10]) is to assume a solution of the form

$$
F(x, y, z)=H(x, y, z) \Psi\left(x^{2}+y^{2}, z\right)
$$

and substitute it into the PWE, yielding

$$
\Psi \nabla_{t}^{2} H+2\left[\vec{\nabla}_{t} H \cdot \vec{\nabla}_{t} \Psi\right]-2 i k H \frac{\partial \Psi}{\partial z}=0 .
$$

The solutions of this equation are assumed to have the form

$$
H(x, y, z)=f\left(\frac{x}{w}\right) g\left(\frac{y}{w}\right) \exp \{-i \Phi(z)\} .
$$

After some manipulations, the following equation results:

$$
\frac{1}{f}\left[\frac{d^{2} f}{d \sigma^{2}}-2 \sigma \frac{d f}{d \sigma}\right]+\frac{1}{g}\left[\frac{d^{2} g}{d \tau^{2}}-2 \tau \frac{d g}{d \tau}\right]-2 k w^{2}(z) \Phi^{\prime}(z)=0,
$$

where $\sigma=\sqrt{2} x / w(z)$ and $\tau=\sqrt{2} y / w(z)$. Bearing in mind that the Hermite polynomials of order $n, h_{n}=h_{n}(\xi)$, satisfy the differential equation $d^{2} h_{n} / d \xi^{2}-2 \xi d h_{n} / d \xi+2 n h_{n}(\xi)=$ 0 , the equation separates into a system of three equations whose solutions are

$$
f_{n}\left(\frac{x}{w}\right)=h_{n}\left(\frac{\sqrt{2} x}{w(z)}\right), g_{m}\left(\frac{y}{w}\right)=h_{m}\left(\frac{\sqrt{2} y}{w(z)}\right), \Phi_{n m}(z)=-(n+m) \arctan \left(\frac{z}{z_{R}}\right) .
$$

Backtracking with these solutions generates the Hermite-Gauss normal modes

$$
\begin{aligned}
& F_{n m}(x, y, z)=v_{n}(x, z) v_{m}(y, z), \\
& v_{n}(x, z)=\sqrt{\frac{w(0)}{w(z)}} e^{-i(n+1 / 2) \psi(z)} h_{n}\left(\frac{\sqrt{2} x}{w(z)}\right) \exp \left\{\phi\left(x^{2}, z\right)\right\},
\end{aligned}
$$

$n, m=0,1,2,3, \ldots$, for the PWE [10, III-28]. The Guoy phase shift is specified by $\psi(z)=\arctan \left(z / z_{R}\right)$. The initial phase angle $\psi_{o}$ is normalized to zero at the focal point $[16$, p. 665].

The normal modes $F_{n m}$ are orthogonal,

$$
\int_{-\infty}^{\infty} \int_{-\infty}^{\infty} F_{n m}(x, y, z) F_{n^{\prime} m^{\prime}}^{*}(x, y, z) d x d y=\gamma_{n m}^{-2} \delta_{n m} \delta_{n^{\prime} m^{\prime}}
$$

in any transverse plane $\left(z=z_{o}\right)$ for $m, n, m^{\prime}, n^{\prime}=0,1,2,3, \ldots$, where $\gamma_{n m}:=\sigma_{n} \sigma_{m}$ and $\sigma_{n}=(2 / \pi)^{1 / 4} / \sqrt{\left(2^{n} n !\right)}$. The orthogonality of the $F_{n m}$ is independent of the $z$-coordinate on account of the fact that energy is conserved during propagation. The superscript "*" designates the complex conjugation of " $i$ " in the definition of $F_{n m}$ and not the variables $(x, y, z)$, which will become independent complex variables later in the development. See 
[16, p. 646-647]. Completeness and decay of the normal modes are discussed in the next section.

3. The integral transforms. We construct solutions of the PWE that are both analytic in the half-space $z>-z_{S}$ and have amplitudes that decay uniformly to zero in the transverse direction as the wave advances along the optical axis. The analysis unlocks by means of dual integral transforms $\left\{T, T^{-1}\right\}$ that link the analytic solutions $F$ with analytic functions $f$ of two complex variables. Properties of the kernels of the transforms allow us to infer these characteristics of $F$.

Consider the normal mode expansion of a solution

$$
\begin{aligned}
& F(x, y, z)=\sum_{n=0}^{\infty} \sum_{m=0}^{\infty} \gamma_{n m} a_{n m} F_{n m}(x, y, z), \\
& F_{n m}(x, y, z)=v_{n}(x, z) v_{m}(y, z)
\end{aligned}
$$

and the associated analytic function

$$
\begin{aligned}
& f(\xi, \eta)=\sum_{n=0}^{\infty} \sum_{m=0}^{\infty} a_{n m} f_{n m}(\xi, \eta), \\
& f_{n m}(\xi, \eta)=\xi^{n} \eta^{m} .
\end{aligned}
$$

The transforms linking these functions are defined in terms of the kernel

$$
K(x, y, z ; \xi, \eta):=J(x, z ; \xi) \cdot J(y, z ; \eta)
$$

where

$$
J(x, z ; \xi):=\sum_{n=0}^{\infty} \sigma_{n} v_{n}(x, z) \xi^{n} .
$$

Concerning the properties of these kernels, let $D_{K}:=\left\{(x, y, z, \xi, \eta):(x, y, z, \xi, \eta) \in E_{o}^{3}\right.$ $\left.\times D^{2}\right\}$ where $E_{o}:=E_{+}^{3} \cup E_{-}^{3}, E_{+}^{3}:=\left\{(x, y, z) \in E^{3}:-z_{S}<z\right\}, E_{-}^{3}:=\{(x, y, z) \in$ $\left.E^{3}: z \leq-z_{S}\right\} /\left\{\left(0,0,-z_{S}\right)\right\}$, and $D^{2}=\left\{(\xi, \eta) \in C^{2}:|\xi|<1,|\eta|<1\right\}$. We now use H. Cramer's estimate [2, p. 208], $\max _{-\infty<x<+\infty}\left\{e^{-x^{2} / 2}\left|h_{n}(x)\right|\right\}<k \sigma_{n}^{-1}$, where $k$ is Charlier's constant, $k=1.0865 \ldots$, for $n=0,1,2,3, \ldots$, to deduce that

$$
\sup _{n \geq 0}\left\{\sigma_{n}\left\{\max _{-z_{R} \leq x<\infty}\left|v_{n}(x, z)\right|\right\}\right\} \leq k \sqrt{w(0) / w(z)}, z \geq 0 .
$$

Thus, the kernel $J(x, z, \xi)$ is analytic on compacta of $D_{K} \cap\{(x, 0, z, \xi, 0):(x, 0, z, \xi, 0) \in$ $\left.E_{o}^{3} \times D^{2}\right\}$. This fact implies that the kernel $K$ is analytic on compacta of the domain $D_{K}$. Moreover, the fact that $\lim _{z \rightarrow \infty}\left\{\sigma_{n}\left|v_{n}(x, z)\right|\right\}=\lim _{z \rightarrow \infty}\left\{\sigma_{n}\left|v_{n}(y, z)\right|\right\}=0$ for all $0 \leq x, y<+\infty$ implies that the normal modes $F_{n m}(x, y, z) \rightarrow 0$ as $z \rightarrow \infty$ in any transverse plane. This property confirms the observation that the normal modes "flatten out", and that the energy density of the component frequencies decreases as their distance from the focal point increases along the optical axis. We conclude that

$$
\lim _{z \rightarrow \infty} K(x, y, z ; \xi, \eta)=0,(x, y, z ; \xi, \eta) \in D_{K} .
$$

Later on we will use this property of the kernel to establish that the profiles of the normal mode expansions of the solutions "flatten out" as well. 
With this information in hand, we proceed to build the requisite transforms. It is easy to verify that

$$
\left(\frac{1}{2 \pi i}\right)^{2} \int_{|\xi|=\epsilon_{1}} \int_{|\eta|=\epsilon_{2}} K(x, y, z ; \xi, \eta) f_{n m}\left(\frac{1}{\xi}, \frac{1}{\eta}\right) \frac{d \xi}{\xi} \frac{d \eta}{\eta}=\gamma_{n m} F_{n m}(x, y, z)
$$

for $\epsilon_{1}, \epsilon_{2}<1$, and

$$
\int_{-\infty}^{\infty} \int_{-\infty}^{\infty} K^{*}(x, y, z ; \xi, \eta) \gamma_{n m} F_{n m}(x, y, z) d x d y=f_{n m}(\xi, \eta)
$$

for $n, m=0,1,2,3, \ldots$, where

$$
K^{*}(x, y, z ; \xi, \eta):=\left[\frac{w(z)}{w(0)}\right]^{2} \sum_{n=0}^{\infty} \sum_{m=0}^{\infty} \gamma_{n m} F_{n m}^{*}(x, y, z) f_{n m}(\xi, \eta)
$$

with $F_{n m}^{*}(x, y, z)=v_{n}^{*}(x, z) v_{m}^{*}(y, z)$ and $\exp \phi^{*}(\xi, z)=\xi\left\{i k / 2 r(z)-1 / w^{2}(z)\right\}$.

Let the associate $f=f(\xi, \eta)$ of $F$ be analytic in the bidisk $D_{\alpha, \beta}^{w} 2=\{(\xi, \eta):|\xi|<$ $|\alpha|,|\eta|<|\beta|\}$ where $1<|\alpha| \leq|\beta|$. The ascending transform is parameterized by $\Gamma_{\alpha}$ : $\xi_{\alpha}^{-1}=\frac{1}{2}[|\alpha|+1] e^{i \theta}$ and $\Gamma_{\beta}: \eta_{\beta}^{-1}=\frac{1}{2}[|\beta|+1] e^{i \varphi}$ for $0 \leq \theta, \varphi<2 \pi$ and defined initially as

$$
F(x, y, z)=T_{o}[f]:=\left(\frac{1}{2 \pi}\right)^{2} \int_{0}^{2 \pi} \int_{0}^{2 \pi} K\left(x, y, z ; \xi_{\alpha}, \eta_{\beta}\right) f\left(\xi_{\alpha}^{-1}, \eta_{\beta}^{-1}\right) d \theta d \varphi .
$$

We observe that because $f$ and $K$ are analytic on the respective domains $D_{\alpha, \beta}^{2}$ and $D_{K}$, $F$ is analytic on $E_{o}^{3}$. Moreover, we infer from the uniform convergence of the kernel $K$ on $D_{K}$ that the $\lim _{z \rightarrow \infty} F(x, y, z)=0$ uniformly in $(x, y)$ for all $(x, y, z) \in E_{+}^{3}$. In the next section, the variables $(x, y, z) \in C^{3}$ and the $\lim _{|z| \rightarrow \infty} F(x, y, z)$ is in general not zero. The initial domain of definition of $F$ is taken as $\{(x, y, z): z>0\}$. This restriction corresponds to beam propagation in the positive $z$-direction from the focal point. The representation of $F$ extends into region $\left\{(x, y, z):-z_{S}<z \leq 0\right\}$. If the associate $f$ is an entire function on $C^{2}$, one may select any pair of complex numbers $\{\alpha, \beta\}$ for which $1<\min \{|\alpha|,|\beta|\}$ and apply the previous reasoning.

Conversely, the integrand of the descending transform

$$
f(\xi, \eta)=T_{o}^{-1}[F]:=\int_{x=-\infty}^{x=+\infty} \int_{y=-\infty}^{y=+\infty} K^{*}(x, y, z ; \xi, \eta) F(x, y, z) d x d y
$$

is analytic for all points $(\xi, \eta) \in D_{K} / E_{+}^{3}$ when $F$ is restricted to its initial domain $E_{+}^{3}$. Thus, $f$ is analytic on $D^{2}$. We have shown that the initial domains of definition of the transforms $T_{o}[f]$ and $T_{o}^{-1}[F]$ are $E_{+}^{3}$ and $D^{2}$ respectively. Consequently the functions $F$ and $f$ are analytic on these domains.

Theorem 1. The integral transform pair $\left\{T_{o}, T_{o}^{-1}\right\}$ forms a unique link between the functions $\{F, f\}$ on their initial domains of definition.

4. The analysis. In this section, we construct a new class of solutions to the PWE in the function theoretic domain. The real variables $(x, y, z)$ are simultaneously allowed to become independent complex variables and the transforms $\left\{T_{o}, T_{o}^{-1}\right\}$ are analytically continued by contour deformation. 
The resulting transforms,

$$
F(x, y, z)=T[f]=\left(\frac{1}{2 \pi i}\right)^{2} \int_{\sigma_{1}} \int_{\sigma_{2}} K(x, y, z ; \xi, \eta) f\left(\frac{1}{\xi}, \frac{1}{\eta}\right) \frac{d \xi}{\xi} \frac{d \eta}{\eta}
$$

and

$$
f(\xi, \eta)=T^{-1}[F]=\int_{\Gamma_{1}} \int_{\Gamma_{2}} K^{*}(x, y, z ; \xi, \eta) F(x, y, z) d x d y
$$

represent function elements on their domains of association (see [6]). Here, $\sigma_{1}$ and $\sigma_{2}$ are simple closed contours homologous to $|\xi|=\left|\xi_{\alpha}\right|$ and $|\eta|=\left|\eta_{\beta}\right|$ modulo the singularities of the integrand of $T[f]$. The reader should note that in $C^{3}$ the domain of association of $F$ also includes $E_{+}^{3}$ as a component of $E_{-}^{3}$. The contours $\Gamma_{1}$ and $\Gamma_{2}$ have end points fixed at infinity relative to the north poles on the product of two of the Riemann spheres $S_{\xi} \times S_{\eta}$ and are homologous to the $x$ and $y$ axes modulo the singularities of the integrand of $T^{-1}[F]$.

Theorem 2. The dual integral transforms $\left\{T, T^{-1}\right\}$ join the unique function elements $\{F, f\}$ on their domains of association.

The new class of solutions of the PWE is constructed by deforming the contours of the ascending operator to include associates

$$
h_{p}(\xi, \alpha) h_{q}(\eta, \beta)
$$

with singularities that are poles of order $(p, q)$ of the form $h_{p}(\xi, \alpha)=1 /(\xi-\alpha)^{p+1}$. Here, the singularities nearest the origin in the $C_{\xi} \times C_{\eta}$ planes are at $(\xi, \eta)=(\alpha, \beta)$, where the $\operatorname{Re}(\alpha)$ and $\operatorname{Re}(\beta)$ are positive and $1<|\alpha| \leq|\beta|$.

If the contour $|\xi|=1$ is first deformed as $\sigma_{1, \alpha}$ to include $\xi=\alpha$, we find that

$$
T_{\sigma_{1, \alpha}}\left[h_{p}(\xi, \alpha)\right]=(-1 / \alpha)^{p+1}(1 / p !)\left[\frac{\partial}{\partial\left[\frac{1}{\alpha}\right]}\right]^{p}\left\{\left(\frac{1}{\alpha}\right)^{p} K\left(x, y, z ; \frac{1}{\alpha}, 0\right)\right\} .
$$

Similarly, inclusion of the singularity in the $C_{\eta}$-plane within the contour $\sigma_{2, \beta}$ produces

$$
T_{\sigma_{2, \beta}}\left[h_{q}(\eta, \beta)\right]=(-1 / \beta)^{q+1}(1 / q !)\left[\frac{\partial}{\partial\left[\frac{1}{\beta}\right]}\right]^{q}\left\{\left(\frac{1}{\beta}\right)^{q} K\left(x, y, z ; 0, \frac{1}{\beta}\right)\right\} .
$$

Inclusion of both singularities then yields

$$
\begin{aligned}
T_{\sigma_{1, \alpha} \sigma_{2, \beta}}\left[h_{p}(\xi, \alpha) h_{q}(\eta, \beta)\right] & =(-1 / \alpha)^{p+1}(-1 / \beta)^{q+1}(1 / p ! q !)\left[\frac{\partial}{\partial\left[\frac{1}{\alpha}\right]}\right]^{p}\left[\frac{\partial}{\partial\left[\frac{1}{\beta}\right]}\right]^{q} \\
& \times\left\{\left(\frac{1}{\alpha}\right)^{p}\left(\frac{1}{\beta}\right)^{q} K\left(x, y, z ; \frac{1}{\alpha}, \frac{1}{\beta}\right)\right\} .
\end{aligned}
$$

Application of the inverse transform to the above expression establishes that the function $h_{p}(\xi, \alpha) h_{q}(\eta, \beta)$ is the associate on the domain of association. We have established the following result for a solution of the PWE whose associate is a rational function.

Theorem 3. Consider the function

$$
f(\xi, \eta)=\sum_{j=1}^{N} \sum_{k=1}^{M} \frac{a_{j k}}{\left(\xi-\alpha_{j}\right)^{p_{j}+1}\left(\eta-\beta_{k}\right)^{q_{k}+1}}
$$


with poles $\left\{\alpha_{1}, \ldots, \alpha_{N} ; \beta_{1}, \ldots, \beta_{M}\right\}$ located in the complement of the closed unit bidisk and in the right half planes of $C_{\xi} \times C_{\eta}$. Then the corresponding function element whose domain of association includes all the poles is

$$
\begin{aligned}
F(x, y, z) & =\sum_{j=1}^{N} \sum_{k=1}^{M} a_{j k}\left(-1 / \alpha_{j}\right)^{p_{j}+1}\left(-1 / \beta_{k}\right)^{q_{k}+1}\left(1 / p_{j} ! q_{k} !\right) \\
& \times\left[\frac{\partial}{\partial\left[\frac{1}{\alpha_{j}}\right]}\right]^{p_{j}}\left[\frac{\partial}{\partial\left[\frac{1}{\beta_{k}}\right]}\right]^{q_{k}}\left\{\left(\frac{1}{\alpha_{j}}\right)^{p_{j}}\left(\frac{1}{\beta_{k}}\right)^{q_{k}} K\left(x, y, z ; \frac{1}{\alpha_{j}}, \frac{1}{\beta_{k}}\right)\right\},
\end{aligned}
$$

and conversely.

The inclusion of a singularity in the domain of association of $f$ results in a jump discontinuity in the function element $F$ by the addition of a sectionally analytic residue element. This element is itself a solution of the PWE in the included component of the extended domain. The norm of the element may be interpreted as an energy differential in the function element $F$ that appears as the appropriate contour crosses the singularity, and the transform of the corresponding residue element is added to $F$. In the absence of source terms in the PWE, the resulting energy differential may be interpreted as the energy radiated by the wave as the pole is crossed. This interpretation dictates limits on the locations and orders of the singularities as the sum total of the radiated energies is limited to that of the original pulse on the unit bidisk. Moreover, the structure of the $T$-operator only allows for the beam to radiate a finite number of times in this model.

It is easy to establish an estimate on this energy as the contour deformations pass through the poles at $(\xi, \eta):=\left(\alpha_{j}, \beta_{k}\right)$. The radiated energy is expressed in terms of the square of the $L_{(E \times E)}^{2}\left(T_{\sigma_{1, \alpha_{j}} \sigma_{2, \beta_{k}}}\left[h_{p_{j}}\left(\xi, \alpha_{j}\right) h_{q_{k}}\left(\eta, \beta_{k}\right)\right]\right)$ energy norm taken over the transverse plane. A lower bound is given by

$$
\begin{aligned}
& \left|a_{j k}\right|^{2}\left|\left(1 / \alpha_{j}\right)^{p_{j}+1}\left(1 / \beta_{k}\right)^{q_{k}+1}\right|^{2}\left(1 / p_{j} ! q_{k} !\right)^{2} \\
& \times \sum_{n=0}^{\infty} \sum_{m=0}^{\infty}\left|\left[\frac{\partial}{\partial\left[\frac{1}{\alpha_{j}}\right]}\right]^{p_{j}}\left[\frac{\partial}{\partial\left[\frac{1}{\beta_{k}}\right]}\right]^{q_{k}}\left\{\left(\frac{1}{\alpha_{j}}\right)^{p_{j}}\left(\frac{1}{\beta_{k}}\right)^{q_{k}} f_{n m}\left(\frac{1}{\alpha_{j}}, \frac{1}{\beta_{k}}\right)\right\}\right|^{2},
\end{aligned}
$$

which simplifies to

$$
\begin{aligned}
& \left|a_{j k}\right|^{2}\left|\left(1 / \alpha_{j}\right)^{p_{j}+1}\left(1 / \beta_{k}\right)^{q_{k}+1}\right|^{2}\left(1 / p_{j} ! q_{k} !\right)^{2} \\
& \times\left|\left[\frac{\partial}{\partial\left[\frac{1}{\alpha_{j}}\right]}\right]^{p_{j}}\left[\frac{\partial}{\partial\left[\frac{1}{\beta_{k}}\right]}\right]^{q_{k}}\left\{\left(\frac{1}{\alpha_{j}}\right)^{p_{j}}\left(\frac{1}{\beta_{k}}\right)^{q_{k}} \frac{1}{\left(1-1 / \alpha_{j}\right)} \frac{1}{\left(1-1 / \beta_{k}\right)}\right\}\right|^{2} .
\end{aligned}
$$

5. Special cases. We begin this section with a study of the analytic solutions in the right half space that are symmetric,

$$
F(x, y, z)=F(y, x, z),
$$

about the plane $y=x$ for $z>-z_{S}$. The series expansion of a symmetric solution and its associate assume particularly simple forms,

$$
F(x, y, z)=\sum_{n=0}^{\infty} \gamma_{n n} a_{n} F_{n n}(x, y, z)
$$


and

$$
f(\xi, \eta)=\sum_{n=0}^{\infty} a_{n} f_{n n}(\xi, \eta)
$$

which allow for sharper results. The ascending and descending kernels are

$$
S(x, y, z ; \xi, \eta):=\sum_{n=0}^{\infty} \gamma_{n n} F_{n n}(x, y, z) f_{n n}(\xi, \eta)
$$

and

$$
S^{*}(x, y, z ; \xi, \eta):=\sum_{n=0}^{\infty} \gamma_{n n} F_{n n}^{*}(x, y, z) f_{n n}(\xi, \eta) .
$$

The reader will note that F. G. Mehler's [1, p. 175, \#12a] generating function

$$
\sum_{n=0}^{\infty} h_{n}(x) h_{n}(y) \frac{(t / 2)^{n}}{n !}=\frac{1}{\left(1-t^{2}\right)^{1 / 2}} \exp \left\{\frac{2 x y-\left(x^{2}+y^{2}\right) t^{2}}{\left(1-t^{2}\right)}\right\}
$$

for the Hermite polynomials allows us to write the kernels in closed form as

$$
\begin{aligned}
S_{+}(x, y, z ; \sqrt{\tau}, \sqrt{\tau}) & =\sqrt{\frac{2}{\pi}} \frac{\left[\frac{w(0)}{w(z)}\right]}{\sqrt{1-\tau^{2}}} \exp \left\{\frac{2}{w(z)}\left[\frac{2 x y-\left(x^{2}+y^{2}\right) \tau^{2}}{\sqrt{1-\tau^{2}}}\right]\right\} \\
& \times e^{-i\left(\psi(z)-\psi_{o}\right)} \exp \left\{\phi\left(x^{2}+y^{2}, z\right)\right\}
\end{aligned}
$$

and

$$
\begin{aligned}
S_{-}^{*}\left(x, y, z ; \sqrt{\tau^{*}}, \sqrt{\tau^{*}}\right) & =\sqrt{\frac{2}{\pi}} \frac{\left[\frac{w(z)}{w(0)}\right]^{2}}{\sqrt{1-\left(\tau^{*}\right)^{2}}} \exp \left\{\frac{2}{w(z)}\left[\frac{2 x y-\left(x^{2}+y^{2}\right)\left(\tau^{*}\right)^{2}}{\sqrt{1-\left(\tau^{*}\right)^{2}}}\right]\right\} \\
& \times e^{i\left(\psi(z)-\psi_{o}\right)} \exp \left\{\phi^{*}\left(x^{2}+y^{2}, z\right)\right\}
\end{aligned}
$$

where $\tau=\tau(\xi, \eta, z)=\xi \eta e^{-2 i \psi(z)}$ and $\tau^{*}=\tau^{*}(\xi, \eta, z)=\xi \eta e^{2 i \psi(z)}$. The complex $C_{\tau}$-plane is cut along $\operatorname{Im}(\tau)=0$ for $\operatorname{Re}(\tau) \geq \pm 1$ and the principal branch is selected.

The resulting integral transforms are

$$
F(x, y, z)=S_{o}[f]:=\left(\frac{1}{2 \pi}\right)^{2} \int_{0}^{2 \pi} \int_{0}^{2 \pi} S_{+}\left(\frac{\sqrt{2} x}{w(z)}, \frac{\sqrt{2} y}{w(z)}, z ; \sqrt{\tau_{\alpha, \beta}}, \sqrt{\tau_{\alpha, \beta}}\right) f\left(\frac{1}{\xi_{\alpha}}, \frac{1}{\eta_{\beta}}\right) d \theta d \varphi
$$

and

$$
f(\xi, \eta)=S_{o}^{-1}[F]:=\int_{x=-\infty}^{x=+\infty} \int_{y=-\infty}^{y=+\infty} S_{-}^{*}\left(\frac{\sqrt{2} x}{w(z)}, \frac{\sqrt{2} y}{w(z)}, z ; \sqrt{\tau^{*}}, \sqrt{\tau^{*}}\right) F(x, y, z) d x d y,
$$

where $\tau_{\alpha, \beta}=\tau\left(\xi_{\alpha}, \eta_{\beta}, z\right)$. We take the initial domain of definition of the kernel as $D_{S}=D_{K}$. Maintaining the same restrictions on the singularities of the associate $f$, we determine that the initial domain of $F$ is $E_{+}^{3}$. The initial domain of $f$ is simply $D_{S} / E_{+}^{3}$.

The real variables $(x, y, z)$ are now allowed to become independent complex variables. The analytic continuation procedure is followed by deforming the contours in the complex plane and avoiding the endpinch singularities at $\tau= \pm 1$ to arrive at the principal branches of the function elements

$$
F(x, y, z)=S[f]:=\left(\frac{1}{2 \pi i}\right)^{2} \int_{\sigma_{1}} \int_{\sigma_{2}} S_{+}\left(\frac{\sqrt{2} x}{w(z)}, \frac{\sqrt{2} y}{w(z)}, z ; \sqrt{\tau}, \sqrt{\tau}\right) f\left(\frac{1}{\xi}, \frac{1}{\eta}\right) \frac{d \xi}{\xi} \frac{d \eta}{\eta}
$$


and

$$
f(\xi, \eta)=S^{-1}[F]:=\int_{\Gamma_{1}} \int_{\Gamma_{2}} S_{-}^{*}\left(\frac{\sqrt{2} x}{w(z)}, \frac{\sqrt{2} y}{w(z)}, z ; \sqrt{\tau^{*}}, \sqrt{\tau^{*}}\right) F(x, y, z) d x d y .
$$

Theorem 4. The dual integral transforms $\left\{S, S^{-1}\right\}$ join the unique function elements $\{F, f\}$ on their domains of association.

Let us determine the jump in $F$ as we deform the contours simultaneously across singularities that are symmetrically placed on the principal branches in the $C_{\xi}$ and $C_{\eta}$ planes at $\alpha_{1, j}=\gamma_{j} e^{i\left(\pi / 4-\theta_{o, j}\right)}$ and $\alpha_{2, j}=\gamma_{j} e^{i\left(\pi / 4+\theta_{o, j}\right)}$ for $0<\theta_{o, j}<\pi / 4$ with $1<\gamma_{1}<$ $\gamma_{2}<\ldots<\gamma_{n}$. We are considering associates of the form

$$
f(\xi, \eta)=\sum_{j=1}^{n} a_{j} f_{j}(\xi, \eta)
$$

where

$$
f_{j}(\xi, \eta)=\frac{1}{\left(\xi-\alpha_{1, j}\right)^{p_{j}+1}\left(\eta-\alpha_{2, j}\right)^{p_{j}+1}}+\frac{1}{\left(\xi-\alpha_{2, j}\right)^{p_{j}+1}\left(\eta-\alpha_{1, j}\right)^{p_{j}+1}} .
$$

The $S$-transform yields

$$
F(x, y, z)=\sum_{j=1}^{n} a_{j} F_{j}(x, y, z)
$$

where for $\mu=\frac{\sqrt{2} x}{w(z)}, v=\frac{\sqrt{2} y}{w(z)}$, and $j=1, \ldots, n$,

$$
\begin{aligned}
& F_{j}(x, y, z)=\left(\frac{1}{\alpha_{1, j} \alpha_{2, j}}\right)^{2 p_{j}+2} \frac{1}{\left(p_{j} !\right)^{2}} \\
& \times\left[\frac{\partial^{2 p_{j}}}{\partial^{p_{j}}\left[\frac{1}{\alpha_{1, j}}\right] \partial^{p_{j}}\left[\frac{1}{\alpha_{2, j}}\right]}\right]\left\{\left(\frac{1}{\alpha_{1, j} \alpha_{2, j}}\right)^{p_{j}} S\left(\mu, v, z ; \tau^{1 / 2}\left(\alpha_{1, j}^{-1}, \alpha_{2, j}^{-1}, z\right), \tau^{1 / 2}\left(\alpha_{1, j}^{-1}, \alpha_{2, j}^{-1}, z\right)\right)\right\} \\
& +\left(\frac{1}{\alpha_{2, j} \alpha_{1, j}}\right)^{2 p_{j}+2} \frac{1}{\left(p_{j} !\right)^{2}} \\
& \times\left[\frac{\partial^{2 p_{j}}}{\partial^{p_{j}}\left[\frac{1}{\alpha_{2, j}}\right] \partial^{p_{j}}\left[\frac{1}{\alpha_{1, j}}\right]}\right]\left\{\left(\frac{1}{\alpha_{2, j} \alpha_{1, j}}\right)^{p_{j}} S\left(\mu, \nu, z ; \tau^{1 / 2}\left(\alpha_{2, j}^{-1}, \alpha_{1, j}^{-1}, z\right), \tau^{1 / 2}\left(\alpha_{2, j}^{-1}, \alpha_{1, j}^{-1}, z\right)\right)\right\} .
\end{aligned}
$$

The energy radiated by the introduction of the term $1 /\left(\xi-\alpha_{1, j}\right)^{p_{j}+1}\left(\eta-\alpha_{2, j}\right)^{p_{j}+1}$ into the domain of association is at least

$$
\left(\frac{1}{\alpha_{1, j} \alpha_{2, j}}\right)^{2 p_{j}+2} \frac{1}{\left(p_{j} !\right)^{2}} \times\left|\left[\frac{\partial^{2 p_{j}}}{\partial^{p_{j}}\left[\frac{1}{\alpha_{1, j}}\right] \partial^{p_{j}}\left[\frac{1}{\alpha_{2, j}}\right]}\right]\left\{\left(\frac{1}{\alpha_{1, j} \alpha_{2, j}}\right)^{p_{j}} \mid \frac{1}{1-\tau^{2}\left(\alpha_{1, j}^{-1}, \alpha_{2, j}^{-1}, 0\right)}\right\}\right|^{2} .
$$

We complete this discussion by considering fields that are independent of the transverse variable $y$, that is, the two-dimensional case where the PWE has the form

$$
\left[\frac{\partial^{2}}{\partial x^{2}}+\frac{\partial^{2}}{\partial z^{2}}-2 i k \frac{\partial}{\partial z}\right] F=0 .
$$

The analytic solutions are expressed by

$$
F(x, z)=\sum_{n=0}^{\infty} \sigma_{n} a_{n} F_{n}(x, z),
$$


where $F_{n}(x, z)=v_{n}(x, z), n=0,1,2, \ldots$, and the corresponding associates are

$$
f(\xi)=\sum_{n=0}^{\infty} a_{n} \xi^{n}
$$

The dual transforms represented by

$$
F(x, z)=R_{o}[f]:=\frac{1}{2 \pi} \sqrt{\frac{w(0)}{w(z)}} \int_{0}^{2 \pi} J\left(x, z ; \xi_{\alpha}\right) f\left(\xi_{\alpha}^{-1}\right) d \theta
$$

and

$$
f(\xi)=R_{o}^{-1}[F]:=\sqrt[3 / 2]{\frac{w(z)}{w(0)}} \int_{-\infty}^{\infty} J^{*}(x, z ; \xi) F(x, z) d x
$$

are valid on their initial domains of definition. Repeating the process of allowing the variables $(x, z)$ to become independent complex variables and analytically continuing the resulting integrals by contour deformation produces the function elements

$$
F(x, z)=R[f]:=\frac{1}{2 \pi i} \sqrt{\frac{w(0)}{w(z)}} \int_{\sigma_{1}} J(x, z ; \xi) f\left(\frac{1}{\xi}\right) \frac{d \xi}{\xi}
$$

and

$$
f(\xi)=R^{-1}[F]:=\sqrt[3 / 2]{\frac{w(z)}{w(0)}} \int_{\Gamma_{1}} J^{*}(x, z ; \xi) F(x, z) d x .
$$

TheOREM 5. The dual integral transforms $\left\{R, R^{-1}\right\}$ join the unique function elements $\{F, f\}$ on their domains of association.

At this point, the analytic continuation of these transforms follows the template established in the earlier analysis. If $h_{p}(\xi, \alpha)=1 /(\xi-\alpha)^{p+1}$, then the function element is

$$
T_{\sigma_{1, \alpha}}\left[h_{p}(\xi, \alpha)\right]=(-1 / \alpha)^{p+1}(1 / p !)\left[\frac{\partial}{\partial\left[\frac{1}{\alpha}\right]}\right]^{p}\left\{\left(\frac{1}{\alpha}\right)^{p} J\left(x, z ; \frac{1}{\alpha}\right)\right\} .
$$

Moreover, the square of the $L^{2}(E)$ energy norm taken over the transverse axis is given by

$$
\left.\left|\left(1 / \alpha_{j}\right)^{p_{j}+1}\left(1 / p_{j} !\right)^{2} \times\right|\left[\frac{\partial}{\partial\left[\frac{1}{\alpha_{j}}\right]}\right]^{p_{j}}\left\{\left(\frac{1}{\alpha_{j}}\right)^{p_{j}} \frac{1}{\left(1-1 / \alpha_{j}\right)}\right\}\right|^{2} .
$$

6. Comments. A primary question of interest is whether the function theoretic residue solutions in $C^{3}$ are manifested in the domain $E_{+}^{3}$ in a way similar to that of evanescent radiation. Moreover, the amount of radiation or norm differential is a function of the order and location of the poles of the associates. Thus, the poles and their orders are limited to certain regions whose geometry is a function of the total energy in the wave. The actual or approximate location of such singularities are of fundamental importance in numerical computations (see [9]). The answer to this question most easily follows from a representation of the kernels in closed form, in which case the exact domains of association can be determined including their intersection with $E_{+}^{3}$. The closed form of the kernels is unknown to the authors. We note that there is an alternative to knowing these closed form expressions as demonstrated in the analysis found in [7]. 
However, such an analysis in the case of the PWE is at least as difficult as summing the kernel in closed form.

Acknowledgment. Partially supported by a grant from the Joint Technology Office.

\section{REFERENCES}

[1] L.C. Andrews, Special Functions for Engineers and Applied Mathematics, MacMillan Publishing Co., New York, 1985. MR779819 (86g:33001)

[2] H. Bateman, Higher Transcendental Functions, vol. 2, Compiled by the Staff of the Bateman Manuscript Proj., McGraw-Hill, New York, 1953.

[3] S. Bergman, Integral Operators in the Theory of Linear Partial Differential Equations, 2nd rev. printing, Springer-Verlag, New York, 1969. MR0239239 (39:596)

[4] H. Begher \& R.P. Gilbert, Transmutations, Transformations and Kernel Functions, Pitman Monographs \& Surveys in Pure and Appl. Math., vols. 58-59, New York, 1992.

[5] W.B. Colson et. al., Laser Handbook: Free Electron Lasers, Elsevier Science LTD, San Diego \& New York, 1991.

[6] D.L. Colton \& R. Kress, Inverse Acoustic and Electromagnetic Scattering, Appl. Math Sci., vol. 93, Springer-Verlag, New York, 1992.

[7] R.P. Gilbert, Function Theoretic Methods in Partial Differential Equations, Math. in Sci. \& Engr., 54, Academic Press, New York, 1969. MR0241789 (39:3127)

[8] R.P. Gilbert \& R.G. Newton (eds.), Analytic Methods in Mathematical Physics, Gordon \& Breach Sci. Pub., New York, 1970. MR0327404 (48:5746)

[9] J. Jin, The Finite Element Method in Electromagnetics, John Wiley \& Sons, New York, 1993. MR1903357 (2004b:78019)

[10] R. Victor Jones, "On Classical Electromagnetic Fields (cont.)", http://people.seas.harvard.edu/ jones/ap216/lectures/ls_1/1s1_u3/1s1_unit_3.html

[11] M. Kracht \& E. O. Kreyszig, Methods of Complex Analysis in Partial Differential Equations with Applications, Can. Math. Soc. Series of Monographs \& Advanced Texts, Wiley-Interscience Publication, New York, 1988. MR941372 (89f:35002)

[12] M.Z. v. Krzywoblocki, Bergman's and Gilbert's operators in elasticity, electromagnetism, fluid dynamics, wave mechanics, Analytic Methods in Mathematical Physics, Gordon \& Breach Scientific Publishers, New York (1970) 207-247.

[13] P.A. McCoy, On Radiating Solutions to the Helmholtz Equation and Inverse Scattering, Appl. Analysis, vol. 77, nos. 3-4 (2001) 319-326. MR1975738 (2004a:35033)

[14] P.A. McCoy, Electromagnetic Field Singularities, J. Math. Anal. \& Appl, vol. 275 (2002), pp. 761-770. MR1943778 (2003m:78014)

[15] C. Muller, Mathematical Theory of Electromagnetic Waves, Die Grundlehren der mathematischen Wissenschaften, rev. ed., vol. 155, Springer-Verlag, New York, 1969. MR0253638 (40:6852)

[16] A.E. Siegman, Lasers, University Science Books, Mill Valley, CA, 1986.

[17] P. Sprangle et. al., Atmospheric Propagation of Ultrashort Laser Pulses, 6th Directed Energy Symposium, Albuquerque, http://www.deps.org/DEPSpages/DEsymp03.html

[18] P. Sprangle et. al., Focusing of Intense Pulses Using Plasma Channels, AIP Conf. Proc., vol. 647(1), pp. 664-673, Dec. 2002.

[19] G. Szego, Orthogonal Polynomials, Amer. Math. Soc. Colloq. Publ., vol. 23, Providence, 1967.

[20] E.T. Whittaker \& G.N. Watson, A Course of Modern Analysis, Cambridge University Press, 4th ed. (reprinted) New York, 1969. MR1424469 (97k:01072) 